\title{
Simple Continued Fractions and Their Convergents
}

\author{
Bo Li \\ Qingdao University of Science \\ and Technology \\ China
}

\author{
Yan Zhang \\ Qingdao University of Science \\ and Technology \\ China
}

\author{
Artur Korniłowicz \\ Institute of Computer Science \\ University of Białystok \\ Sosnowa 64, 15-887 Białystok, Poland
}

Summary. The article introduces simple continued fractions. They are defined as an infinite sequence of integers. The characterization of rational numbers in terms of simple continued fractions is shown. We also give definitions of convergents of continued fractions, and several important properties of simple continued fractions and their convergents.

MML identifier: REAL_3, version: 7.8.01 4.70.946

The articles [15], [6], [18], [4], [2], [13], [3], [7], [8], [16], [17], [1], [19], [20], [14], $[10],[5],[9],[11]$, and [12] provide the notation and terminology for this paper.

\section{Preliminaries}

For simplicity, we adopt the following convention: $a, b, k, n, m$ are natural numbers, $i$ is an integer, $r$ is a real number, $p$ is a rational number, $c$ is a complex number, $x$ is a set, and $f$ is a function.

Let us consider $n$. One can check the following observations:

* $n \div 0$ is zero,

* $n \bmod 0$ is zero, 
* $0 \div n$ is zero, and

* $0 \bmod n$ is zero.

Let us consider $c$. One can verify that $c-c$ is zero and $\frac{c}{0}$ is zero.

Let us note that $\lfloor 0\rfloor$ is zero.

The following propositions are true:

(1) If $0<r$ and $r<1$, then $1<\frac{1}{r}$.

(2) If $i \leq r$ and $r<i+1$, then $\lfloor r\rfloor=i$.

(3) $\left\lfloor\frac{m}{n}\right\rfloor=m \div n$.

(4) If $m \bmod n=0$, then $\frac{m}{n}=m \div n$.

(5) If $\frac{m}{n}=m \div n$, then $m \bmod n=0$.

(6) $\operatorname{frac}\left(\frac{m}{n}\right)=\frac{m \bmod n}{n}$.

(7) If $p \geq 0$, then there exist natural numbers $m, n$ such that $n \neq 0$ and $p=\frac{m}{n}$.

Let $R$ be a binary relation. We say that $R$ is integer-yielding if and only if: (Def. 1) $\operatorname{rng} R \subseteq \mathbb{Z}$.

One can verify that every binary relation which is natural-yielding is also integer-yielding.

One can check the following observations:

* there exists a function which is natural-yielding,

* every binary relation which is empty is also integer-yielding, and

* every binary relation which is integer-yielding is also real-yielding.

Let $D$ be a set. One can verify that every partial function from $D$ to $\mathbb{Z}$ is integer-yielding.

Let $f$ be an integer-yielding function and let $n$ be a set. One can verify that $f(n)$ is integer.

Let us note that there exists a sequence of real numbers which is integeryielding.

An integer sequence is an integer-yielding sequence of real numbers.

One can prove the following proposition

(8) $\quad f$ is an integer sequence iff $\operatorname{dom} f=\mathbb{N}$ and for every $x$ such that $x \in \mathbb{N}$ holds $f(x)$ is integer.

Let $f$ be a natural-yielding function and let $n$ be a set. Note that $f(n)$ is natural.

We now state three propositions:

(9) $\quad f$ is a function from $\mathbb{N}$ into $\mathbb{Z}$ iff $f$ is an integer sequence.

(10) $\quad f$ is a sequence of naturals iff $\operatorname{dom} f=\mathbb{N}$ and for every $x$ such that $x \in \mathbb{N}$ holds $f(x)$ is natural.

(11) $\quad f$ is a function from $\mathbb{N}$ into $\mathbb{N}$ iff $f$ is a sequence of naturals. 


\section{On the Euclidean Algorithm}

Let $m, n$ be natural numbers. The functor $\operatorname{modSeq}(m, n)$ yielding a sequence of naturals is defined by:

$($ Def. 2) $(\operatorname{modSeq}(m, n))(0)=m \bmod n$ and $(\operatorname{modSeq}(m, n))(1)=n \bmod (m \bmod$ $n$ ) and for every natural number $k$ holds $(\operatorname{modSeq}(m, n))(k+2)=$ $(\operatorname{modSeq}(m, n))(k) \bmod (\operatorname{modSeq}(m, n))(k+1)$.

Let $m, n$ be natural numbers. The functor $\operatorname{divSeq}(m, n)$ yielding a sequence of naturals is defined as follows:

(Def. 3) $(\operatorname{divSeq}(m, n))(0)=m \div n$ and $(\operatorname{divSeq}(m, n))(1)=n \div(m \bmod$ $n$ ) and for every natural number $k$ holds $(\operatorname{divSeq}(m, n))(k+2)=$ $(\operatorname{modSeq}(m, n))(k) \div(\operatorname{modSeq}(m, n))(k+1)$.

We now state several propositions:

(12) $(\operatorname{divSeq}(m, n))(1)=n \div(\operatorname{modSeq}(m, n))(0)$.

(13) $(\operatorname{modSeq}(m, n))(1)=n \bmod (\operatorname{modSeq}(m, n))(0)$.

(14) If $a \leq b$ and $(\operatorname{modSeq}(m, n))(a)=0$, then $(\operatorname{modSeq}(m, n))(b)=0$.

(15) If $a<b$, then $(\operatorname{modSeq}(m, n))(a)>(\operatorname{modSeq}(m, n))(b)$ or $(\operatorname{modSeq}(m, n))(a)=0$.

(16) If $(\operatorname{divSeq}(m, n))(a+1)=0$, then $(\operatorname{modSeq}(m, n))(a)=0$.

(17) If $a \neq 0$ and $a \leq b$ and $(\operatorname{divSeq}(m, n))(a)=0$, then $(\operatorname{divSeq}(m, n))(b)=$ 0.

(18) If $a<b$ and $(\operatorname{modSeq}(m, n))(a)=0$, then $(\operatorname{divSeq}(m, n))(b)=0$.

(19) If $n \neq 0$, then $m=(\operatorname{divSeq}(m, n))(0) \cdot n+(\operatorname{modSeq}(m, n))(0)$.

(20) If $n \neq 0$, then $\frac{m}{n}=(\operatorname{divSeq}(m, n))(0)+\frac{1}{(\operatorname{modSeq}(m, n))(0)}$.

One can prove the following propositions:

(21) $\operatorname{divSeq}(m, 0)=\mathbb{N} \longmapsto 0$.

(22) $\operatorname{modSeq}(m, 0)=\mathbb{N} \longmapsto 0$.

(23) $\operatorname{divSeq}(0, n)=\mathbb{N} \longmapsto 0$.

(24) $\operatorname{modSeq}(0, n)=\mathbb{N} \longmapsto 0$.

(25) There exists a natural number $k$ such that $(\operatorname{divSeq}(m, n))(k)=0$ and $(\operatorname{modSeq}(m, n))(k)=0$.

\section{Simple Continued Fractions}

Let $r$ be a real number. The remainders for s.c.f. of $r$ yields a sequence of real numbers and is defined by the conditions (Def. 4).

(Def. 4)(i) (The remainders for s.c.f. of $r$ ) $(0)=r$, and

(ii) for every natural number $n$ holds (the remainders for s.c.f. of $r)(n+1)=$ $\frac{1}{\text { frac (the remainders for s.c.f. of } r)(n)}$. 
Let $r$ be a real number. We introduce $\operatorname{rfs} r$ as a synonym of the remainders for s.c.f. of $r$.

Let $r$ be a real number. The simple continued fraction of $r$ yielding an integer sequence is defined by:

(Def. 5) For every natural number $n$ holds (the simple continued fraction of $r)(n)=\lfloor(\operatorname{rfs} r)(n)\rfloor$.

Let $r$ be a real number. We introduce scf $r$ as a synonym of the simple continued fraction of $r$.

The following propositions are true:

(26) $(\operatorname{rfs} r)(n+1)=\frac{1}{(\operatorname{rfs} r)(n)-(\operatorname{scf} r)(n)}$.

(27) If $(\operatorname{rfs} r)(n)=0$ and $n \leq m$, then $(\operatorname{rfs} r)(m)=0$.

(28) If $(\operatorname{rfs} r)(n)=0$ and $n \leq m$, then $(\operatorname{scf} r)(m)=0$.

(29) $\quad(\operatorname{rfs} i)(n+1)=0$.

(30) $\quad(\operatorname{scf} i)(0)=i$ and $(\operatorname{scf} i)(n+1)=0$.

(31) If $i>1$, then $\left(\operatorname{rfs}\left(\frac{1}{i}\right)\right)(1)=i$ and $\left(\operatorname{rfs}\left(\frac{1}{i}\right)\right)(n+2)=0$.

(32) If $i>1$, then $\left(\operatorname{scf}\left(\frac{1}{i}\right)\right)(0)=0$ and $\left(\operatorname{scf}\left(\frac{1}{i}\right)\right)(1)=i$ and $\left(\operatorname{scf}\left(\frac{1}{i}\right)\right)(n+2)=0$.

(33) If for every $n$ holds $(\operatorname{scf} r)(n)=0$, then $(\operatorname{rfs} r)(n)=0$.

(34) If for every $n$ holds $(\operatorname{scf} r)(n)=0$, then $r=0$.

(35) $\quad$ frac $r=r-(\operatorname{scf} r)(0)$.

(36) $(\operatorname{rfs} r)(n+1)=\left(\operatorname{rfs}\left(\frac{1}{\operatorname{frac} r}\right)\right)(n)$.

(37) $(\operatorname{scf} r)(n+1)=\left(\operatorname{scf}\left(\frac{1}{\operatorname{frac} r}\right)\right)(n)$.

(38) If $n \geq 1$, then $(\operatorname{scf} r)(n) \geq 0$.

(39) If $n \geq 1$, then $(\operatorname{scf} r)(n) \in \mathbb{N}$.

(40) If $n \geq 1$ and $(\operatorname{scf} r)(n) \neq 0$, then $(\operatorname{scf} r)(n) \geq 1$.

(41) $\left(\operatorname{scf}\left(\frac{m}{n}\right)\right)(k)=(\operatorname{divSeq}(m, n))(k)$ and $\left(\operatorname{rfs}\left(\frac{m}{n}\right)\right)(1)=\frac{n}{(\operatorname{modSeq}(m, n))(0)}$ and $\left(\operatorname{rfs}\left(\frac{m}{n}\right)\right)(k+2)=\frac{(\operatorname{modSeq}(m, n))(k)}{(\operatorname{modSeq}(m, n))(k+1)}$.

(42) $\quad r$ is rational iff there exists $n$ such that for every $m$ such that $m \geq n$ holds $(\operatorname{scf} r)(m)=0$.

(43) If for every $n$ holds $(\operatorname{scf} r)(n) \neq 0$, then $r$ is irrational.

\section{Convergents of Simple Continued Fractions}

In the sequel $n_{1}, n_{2}$ are natural numbers.

Let $r$ be a real number. The convergent numerators of $r$ yielding a sequence of real numbers is defined by the conditions (Def. 6).

(Def. 6)(i) (The convergent numerators of $r)(0)=(\operatorname{scf} r)(0)$,

(ii) (the convergent numerators of $r)(1)=(\operatorname{scf} r)(1) \cdot(\operatorname{scf} r)(0)+1$, and 
(iii) for every natural number $n$ holds (the convergent numerators of $r)(n+$ $2)=(\operatorname{scf} r)(n+2) \cdot($ the convergent numerators of $r)(n+1)+($ the convergent numerators of $r)(n)$.

Let $r$ be a real number. The convergent denominators of $r$ yields a sequence of real numbers and is defined by the conditions (Def. 7).

(Def. 7)(i) (The convergent denominators of $r)(0)=1$,

(ii) (the convergent denominators of $r)(1)=(\operatorname{scf} r)(1)$, and

(iii) for every natural number $n$ holds (the convergent denominators of $r)(n+2)=(\operatorname{scf} r)(n+2) \cdot($ the convergent denominators of $r)(n+1)+($ the convergent denominators of $r)(n)$.

Let $r$ be a real number. We introduce $c n r$ as a synonym of the convergent numerators of $r$. We introduce $c d r$ as a synonym of the convergent denominators of $r$.

One can prove the following propositions:

(44) If $(\operatorname{scf} r)(0)>0$, then for every $n$ holds $(c n r)(n) \in \mathbb{N}$.

(45) If $(\operatorname{scf} r)(0)>0$, then for every $n$ holds $(c n r)(n)>0$.

(46) If $(\operatorname{scf} r)(0)>0$, then for every $n$ holds $(c n r)(n+2)>(\operatorname{scf} r)(n+2)$. $($ cn $r)(n+1)$.

(47) If $(\operatorname{scf} r)(0)>0$, then for every $n$ such that $n_{1}=(c n r)(n+1)$ and $n_{2}=(c n r)(n)$ holds $\operatorname{gcd}\left(n_{1}, n_{2}\right)=1$.

(48) If $(\operatorname{scf} r)(0)>0$ and for every $n$ holds $(\operatorname{scf} r)(n) \neq 0$, then for every $n$ holds $($ cn $r)(n) \geq \tau^{n}$.

(49) If $(\operatorname{scf} r)(0)>0$ and for every $n$ holds $(\operatorname{scf} r)(n) \leq b$, then for every $n$ holds $($ cn $r)(n) \leq\left(\frac{b+\sqrt{b^{2}+4}}{2}\right)^{n+1}$.

(50) $(c d r)(n) \in \mathbb{N}$.

(51) $\quad(c d r)(n) \geq 0$.

(52) If $(\operatorname{scf} r)(1)>0$, then for every $n$ holds $(c d r)(n)>0$.

(53) $(c d r)(n+2) \geq(\operatorname{scf} r)(n+2) \cdot(c d r)(n+1)$.

(54) If $(\operatorname{scf} r)(1)>0$, then for every $n$ holds $(c d r)(n+2)>(\operatorname{scf} r)(n+2)$. $(c d r)(n+1)$.

(55) If for every $n$ holds $(\operatorname{scf} r)(n)>0$, then for every $n$ such that $n \geq 1$ holds $\frac{1}{(c d r)(n) \cdot(c d r)(n+1)}<\frac{1}{(\operatorname{scf} r)(n+1) \cdot(c d r)(n)^{2}}$.

(56) If for every $n$ holds $(\operatorname{scf} r)(n) \leq b$, then for every $n$ holds $(c d r)(n+1) \leq$ $\left(\frac{b+\sqrt{b^{2}+4}}{2}\right)^{n+1}$.

(57) If $n_{1}=(c d r)(n+1)$ and $n_{2}=(c d r)(n)$, then $\operatorname{gcd}\left(n_{1}, n_{2}\right)=1$.

(58) If for every $n$ holds $(\operatorname{scf} r)(n)>0$, then for every $n$ holds $\frac{(c d r)(n+1)}{(c d r)(n)} \geq$ $\frac{1}{(\operatorname{scf} r)(n+2)}$. 
(59) If for every $n$ holds $(\operatorname{scf} r)(n)>0$, then for every $n$ holds $(c d r)(n+2) \leq$ $2 \cdot(\operatorname{scf} r)(n+2) \cdot(c d r)(n+1)$.

(60) If for every $n$ holds $(\operatorname{scf} r)(n) \neq 0$, then for every $n$ holds $\frac{1}{(\operatorname{scf} r)(n+1) \cdot(c d r)(n)^{2}} \leq \frac{1}{(c d r)(n)^{2}}$.

(61) If for every $n$ holds $(\operatorname{scf} r)(n) \neq 0$, then for every $n$ holds $(c d r)(n+1) \geq$ $\tau^{n}$.

(62) If $a>0$ and for every $n$ holds $(\operatorname{scf} r)(n) \geq a$, then for every $n$ holds $(c d r)(n+1) \geq\left(\frac{a+\sqrt{a^{2}+4}}{2}\right)^{n}$.

(63) $\frac{(c n r)(n+2)}{(c d r)(n+2)}=\frac{(\operatorname{scf} r)(n+2) \cdot(c n r)(n+1)+(c n r)(n)}{(\operatorname{scf} r)(n+2) \cdot(c d r)(n+1)+(c d r)(n)}$.

(64) $(c n r)(n+1) \cdot(c d r)(n)-(c n r)(n) \cdot(c d r)(n+1)=(-1)^{n}$.

(65) If for every $n$ holds $(c d r)(n) \neq 0$, then $\frac{(c n r)(n+1)}{(c d r)(n+1)}-\frac{(c n r)(n)}{(c d r)(n)}=$ $\frac{(-1)^{n}}{(c d r)(n+1) \cdot(c d r)(n)}$.

(66) $(c n r)(n+2) \cdot(c d r)(n)-(c n r)(n) \cdot(c d r)(n+2)=(-1)^{n} \cdot(\operatorname{scf} r)(n+2)$.

(67) If for every $n$ holds $(c d r)(n) \neq 0$, then $\frac{(c n r)(n+2)}{(c d r)(n+2)}-\frac{(c n r)(n)}{(c d r)(n)}=$ $\frac{(-1)^{n} \cdot(\operatorname{scf} r)(n+2)}{(c d r)(n+2) \cdot(c d r)(n)}$.

(68) If for every $n$ holds $(\operatorname{scf} r)(n) \neq 0$, then for every $n$ such that $n \geq 1$ holds $\frac{(c n r)(n)}{(c d r)(n)}=\frac{(c n r)(n+1)-(c n r)(n-1)}{(c d r)(n+1)-(c d r)(n-1)}$.

(69) If for every $n$ holds $(c d r)(n) \neq 0$, then for every $n$ holds $\mid \frac{(c n r)(n+1)}{(c d r)(n+1)}-$ $\frac{(c n r)(n)}{(c d r)(n)} \mid=\frac{1}{|(c d r)(n+1) \cdot(c d r)(n)|}$.

(70) If $(\operatorname{scf} r)(1)>0$, then for every $n$ holds $\frac{(c n r)(2 \cdot n+1)}{(c d r)(2 \cdot n+1)}>\frac{(c n r)(2 \cdot n)}{(c d r)(2 \cdot n)}$.

Let $r$ be a real number. The convergents of continued fractions of $r$ yielding a sequence of real numbers is defined as follows:

(Def. 8) The convergents of continued fractions of $r=c n r / c d r$.

Let $r$ be a real number. We introduce $\operatorname{cocf} r$ as a synonym of the convergents of continued fractions of $r$.

One can prove the following propositions:

(71) $(\operatorname{cocf} r)(0)=(\operatorname{scf} r)(0)$.

(72) If $(\operatorname{scf} r)(1) \neq 0$, then $(\operatorname{cocf} r)(1)=(\operatorname{scf} r)(0)+\frac{1}{(\operatorname{scf} r)(1)}$.

(73) If for every $n$ holds $(\operatorname{scf} r)(n)>0$, then $(\operatorname{cocf} r)(2)=(\operatorname{scf} r)(0)+$ $\frac{1}{(\operatorname{scf} r)(1)+\frac{1}{(\operatorname{scf} r)(2)}}$.

(74) If for every $n$ holds $(\operatorname{scf} r)(n)>0$, then $(\operatorname{cocf} r)(3)=(\operatorname{scf} r)(0)+$ $\frac{1}{(\operatorname{scf} r)(1)+\frac{1}{(\operatorname{scf} r)(2)+\frac{1}{(\operatorname{scf} r)(3)}}}$.

(75) If for every $n$ holds $(\operatorname{scf} r)(n)>0$, then for every $n$ such that $n \geq 1$ holds $\frac{(c n r)(2 \cdot n+1)}{(c d r)(2 \cdot n+1)}<\frac{(c n r)(2 \cdot n-1)}{(c d r)(2 \cdot n-1)}$. 
(76) If for every $n$ holds $(\operatorname{scf} r)(n)>0$, then for every $n$ such that $n \geq 1$ holds $\frac{(c n r)(2 \cdot n)}{(c d r)(2 \cdot n)}>\frac{(c n r)(2 \cdot n-2)}{(c d r)(2 \cdot n-2)}$.

(77) If for every $n$ holds $(\operatorname{scf} r)(n)>0$, then for every $n$ such that $n \geq 1$ holds $\frac{(c n r)(2 \cdot n)}{(c d r)(2 \cdot n)}<\frac{(c n r)(2 \cdot n-1)}{(c d r)(2 \cdot n-1)}$

Let $r$ be a real number. The back continued fraction of $r$ yields a sequence of real numbers and is defined by the conditions (Def. 9).

(Def. 9)(i) (The back continued fraction of $r)(0)=(\operatorname{scf} r)(0)$, and

(ii) for every natural number $n$ holds (the back continued fraction of $r)(n+$ 1) $=\frac{1}{(\text { the back continued fraction of } r)(n)}+(\operatorname{scf} r)(n+1)$.

Let $r$ be a real number. We introduce bcf $r$ as a synonym of the back continued fraction of $r$.

One can prove the following proposition

(78) If $(\operatorname{scf} r)(0)>0$, then for every $n$ holds $(\operatorname{bcf} r)(n+1)=\frac{(c n r)(n+1)}{(c n r)(n)}$.

\section{REFERENCES}

[1] Grzegorz Bancerek. The fundamental properties of natural numbers. Formalized Mathematics, 1(1):41-46, 1990.

[2] Grzegorz Bancerek. The ordinal numbers. Formalized Mathematics, 1(1):91-96, 1990.

[3] Czesław Byliński. The complex numbers. Formalized Mathematics, 1(3):507-513, 1990.

[4] Czesław Byliński. Functions and their basic properties. Formalized Mathematics, 1(1):5565, 1990.

[5] Czesław Byliński. Functions from a set to a set. Formalized Mathematics, 1(1):153-164, 1990.

[6] Czesław Byliński. Some basic properties of sets. Formalized Mathematics, 1(1):47-53, 1990.

[7] Krzysztof Hryniewiecki. Basic properties of real numbers. Formalized Mathematics, 1(1):35-40, 1990.

[8] Andrzej Kondracki. Basic properties of rational numbers. Formalized Mathematics, $1(5): 841-845,1990$.

[9] Jarosław Kotowicz. Monotone real sequences. Subsequences. Formalized Mathematics, $1(3): 471-475,1990$.

[10] Jarosław Kotowicz. Real sequences and basic operations on them. Formalized Mathematics, 1(2):269-272, 1990.

[11] Rafał Kwiatek. Factorial and Newton coefficients. Formalized Mathematics, 1(5):887-890, 1990.

[12] Robert M. Solovay. Fibonacci numbers. Formalized Mathematics, 10(2):81-83, 2002.

[13] Andrzej Trybulec. Subsets of complex numbers. To appear in Formalized Mathematics.

[14] Andrzej Trybulec. Binary operations applied to functions. Formalized Mathematics, 1(2):329-334, 1990.

[15] Andrzej Trybulec. Tarski Grothendieck set theory. Formalized Mathematics, 1(1):9-11, 1990.

[16] Andrzej Trybulec and Czesław Byliński. Some properties of real numbers. Formalized Mathematics, 1(3):445-449, 1990.

[17] Michał J. Trybulec. Integers. Formalized Mathematics, 1(3):501-505, 1990.

[18] Zinaida Trybulec. Properties of subsets. Formalized Mathematics, 1(1):67-71, 1990.

[19] Edmund Woronowicz. Relations and their basic properties. Formalized Mathematics, 1(1):73-83, 1990. 
[20] Edmund Woronowicz. Relations defined on sets. Formalized Mathematics, 1(1):181-186, 1990.

Received August 18, 2006 